\title{
La distribución de la superficie de viñedo en Castilla y León según sus variables topográficas
}

\author{
Julio FERNÁNDEZ PoRTELA \\ Departamento de Geografía. \\ Universidad de Valladolid \\ jfportela@geo.uva.es
}

Recibido: 21 de abril del 2014

Enviado a evaluar: 8 de mayo del 2014

Aceptado: 24 de junio del 2014

\section{RESUMEN}

Castilla y León es una región con un peso de la actividad agraria muy importante siendo el viñedo uno de los cultivos más representativos de este territorio, pero sobre todo, de las llanuras centrales de la cuenca del Duero. Las variables topográficas, y en concreto, la altitud y la pendiente, y en menor medida la exposición, van a determinar la distribución de los viñedos por el territorio de esta región. Para la elaboración de esta investigación se va a utilizar el Modelo Digital del Terreno de Castilla y León y los datos de superficie de vides proporcionados por el Sistema de Información Sobre Ocupación de Suelo en España (2005), y a través de los Sistemas de Información Geográfica, utilizando el programa ArcGIS 10.1, y la hoja de cálculo Excel, se van a elaborar un conjunto de mapas y gráficos que permitan ver la distribución de este cultivo por este espacio.

Palabras clave: Superficie de viñedo, variables topográficas, altitud, pendiente, orientación, Castilla y León.

\section{Distribution of surface vineyard in Castilla y León by their topographic variables}

\begin{abstract}
Castilla y León is a region with a weight of important agricultural activity and the vineyard one of the most representative crops of this region, but especially in the central plains of the River Duero. Topographic variables, and in particular the altitude and slope, and less exposure, will determine the distribution of the vineyards in the territory of the region. For the preparation of this paper is to use the Digital Terrain Model of Castilla y León and surface data vines provided by the Information System on Occupation of Land in Spain (2005), and through the GIS , using the ArcGIS 10.1 program and the Excel spreadsheet, that will allow to develop a set of maps and graphics that let you see the distribution of this crop in this area.
\end{abstract}

Key words: Vineyard area, topographic variables, elevation, slope, exposure, Castilla y León. 


\section{Distribution de surface vignoble en Castilla y León par leurs variables topographiques}

\section{RÉSUMÉ}

Castilla y León est une région avec un poids de l'activité agricole important et la vigne une des cultures les plus représentatifs de cette région, mais surtout dans les plaines centrales de la rivière Duero. Variables topographiques, et en particulier de l'altitude et de la pente, et moins d'exposition, permettra de déterminer la répartition des vignes sur le territoire de la région. Pour la préparation de cette recherche est d'utiliser le modèle numérique de terrain de Castille et León et vignes de données de surface fournies par le Système d'information sur l'occupation des terres en Espagne (2005), et par le SIG, en utilisant le programme ArcGIS 10.1, et la feuille de calcul Excel, ils mettront au point un ensemble de cartes et de graphiques qui vous permettent de voir la répartition de cette culture dans ce domaine

Mots clés: Zone de vignobles, variables topographiques, altitude, pente, exposition, Castilla y León.

\section{INTRODUCCIÓN}

El posible origen de la viticultura en la actual región de Castilla y León se remonta a la llegada de los vacceos en torno a los siglos VII y V a.C. según algunos de los restos arqueológicos encontrados en el Yacimiento de Pintia en la localidad vallisoletana de Padilla de Duero (perteneciente al municipio de Peñafiel), y corazón de la Denominación de Origen más emblemática de la comunidad autónoma como es la Ribera del Duero (Sanz et al. 2009). Cómo indicios más representativos de ello se encuentran los restos de cerámica hallados en algunos ajuares, y en cuyo interior han aparecido una serie de sustancias como son los tartratos, ácido cerótico y glucosa, todos ellos elementos contenidos en el vino, hecho que posibilita el consumo del mismo entre estos pobladores del valle central del río Duero.

Junto a este sector, y unos siglos más tarde, en concreto en el siglo III a.C., hay que mencionar otro ejemplo muy relevante que contribuye a consolidar la idea del consumo de este caldo y la producción de uva en las inmediciones del Duero, como revela el descubrimiento de pepitas de uva entre restos de vasijas y copas en algunas excavaciones de Cauca, actual Coca en la provincia de Segovia (Blanco, 2009). Todo ello evidencias, que, a pesar de todo, no son suficientes para afirmar el cultivo y la vinificación de las vides en este espacio de una forma categórica.

Es a partir de la llegada de los romanos, pero sobre todo con el proceso repoblador tras la Reconquista, cuando el viñedo comienza a expandirse por la región como un cultivo clave que ayude a asentar nueva población en las bastas llanuras de Castilla, las cuales habían quedado desiertas como consecuencia de las continuas incursiones y luchas entre cristianos y musulmanes convirtiendo a este espacio en una especie de "Tierra de nadie" (Huetz, 1967; Valdeón, 2006).

Las vides se fueron asentando por el territorio aprovechando todo tipo de suelos experimentando una considerable expansión a lo largo de los siglos, alcanzando su cenit con la invasión de la filoxera en Francia, la cual arrasó prácticamente toda la superficie del país vecino, y este se vio obligado a importar vino español. Los 
viticultores y bodegueros españoles vieron un negocio en el ataque del parásito al campo francés, y se produjo una fiebre de las plantaciones aumentándolas en alrededor de un millón de nuevas hectáreas, pasando aproximadamente de 1,4 millones de hectáreas en 1880, a los 2,4 millones a finales del siglo XIX (Camarero, 1984), un crecimiento desmesurado que se produjo con una escasa planificación sobre el territorio y sin tener en cuenta las posibles consecuencias del futuro. Repercusiones que no tardaron en llegar y que provocaron grandes estragos en los viñedos de prácticamente toda España.

Esta época dorada del viñedo español vio su final con la entrada del parásito en la Península Ibérica, el cual tuvo los mismos efectos devastadores y redujo la superficie de vides provocando importantes pérdidas económicas en las comarcas vitivinicolas españolas (Solano, 1991; Montoya, 2012). Este hito marco un antes y un después en la historia agraria de España, y en concreto en la de Castilla y León. Si además sumamos la importante crisis del medio rural de mediados del siglo XX como consecuencia de la puesta en marcha de una serie de políticas como la concentración parcelaria (Alario, 1991), la expansión del regadío (Eugenio, 2006) o la mecanización (Arias, 2000), se obtiene como resultado un descenso de hectáreas de viñedo en esta región muy significativo pasando de las 290.333 en 1889 (Piqueras, 2005) a las 74.841 existentes en 2010 (Consejería de Agricultura y Ganadería de la Junta de Castilla y León). Una disminución muy considerable de la superficie, pero que se ha adaptando a las nuevas exigencias del mercado y a la coyuntura económica de este sector orientándose hacia la producción de caldos de calidad amparados bajo diversas figuras de protección como la Denominación de Origen, la Indicación Geográfica Protegida o los Vinos de Calidad, y que al mismo tiempo han introducido significativas transformaciones en el paisaje del viñedo tradicional de Castilla y León (Molinero, 2011).

Por lo tanto, la viticultura es una actividad que se lleva realizando desde hace siglos en esta región y la distribución de la superficie de viñedo ha ido variando con el paso de los años dependiendo de la situación económica, política y social existente en estas tierras. En situaciones de expansión ha aprovechado todo tipo de formas de relieve, es decir, terrazas, glacis, laderas, etc., en suelos con aptitudes agronómicas muy buenas frente a otros no tan buenas, pero siempre dentro de unos límites altitudinales que condicionan el desarrollo vegetativo de este tipo de plantas, y con unas pendientes no muy elevadas como consecuencia de la planitud típica de las tierras castellanas.

Teniendo en cuenta lo comentado en las líneas anteriores, el objetivo de esta investigación pretende conocer la distribución espacial actual de la superficie de viñedo existente en Castilla y León en función de una serie de variables topográficas como son la altitud, la pendiente y la orientación. Factores que van a resultar claves para la obtención de una uva de calidad junto con el clima y los suelos, además de las técnicas de laboreo empleadas en el terruño, y posteriormente los métodos y herramientas manejadas en el proceso de elaboración del vino. 


\section{2. ÁREA DE ESTUDIO: EL CLIMA Y LOS SUELOS COMO FACTORES CLAVE EN EL CULTIVO DEL VIÑEDO DE CASTILLA Y LEÓN}

Castilla y León se encuentra rodeada por un cíngulo montañoso compuesto por diferentes cordilleras como son la Cantábrica al norte, la Ibérica al este y la Central al sur con altitudes en determinados sectores superiores a los 2.500 metros. Por último hay que mencionar las penillanuras del oeste que se encargan de delimitar el territorio español del portugués donde el río Duero se va a encajonar ente los materiales graníticos característicos de este espacio. Frente a este reborde montañoso hay que destacar las grandes llanuras del interior con una altitud media elevada de unos 600 metros, superando los 900 en determinados sectores.

Esta diferencia en el relieve ha sido descrita por Jesús García Fernández (1986), en cuya obra El clima en Castilla y León, marcaba esta dualidad entre las llanuras y las montañas haciendo referencia a La Castilla de las llanuras y las montañas de la Castilla. Esta disposición del relieve da lugar a que el clima sea muy contrastado entre una u otra unidad, pero al mismo tiempo existen relaciones entre ambos elementos hasta tal punto que las montañas son uno de los factores claves que lo definen en las llanuras "El relieve se convierte de esta manera en un factor fundamental del clima de Castilla y León, pues es el que motiva, o bien matiza, sus principales rasgos" (Morales y Ortega, 2002: 387).

Por lo tanto, el enclaustramiento de la cuenca por el borde montañoso y su elevada altitud, son los dos principales factores que marcan el clima de la región, el cual va a ser trascendental a la hora de la distribución del viñedo en determinados territorios estableciendo el límite de la planta a una determinada altitud, a partir de la cual, la vid encontraría serias dificultades para sobrevivir, sobre todo debido al alargamiento del periodo libre de heladas, extendiéndose más allá del mes de mayo, así como adelantándose a septiembre, lo que sería perjudicial para el fruto en su periodo de floración y final de su madurez respectivamente. Estos componentes provocan pocas precipitaciones y un descenso de las temperaturas en el invierno, ya que este cíngulo montañoso actúa como una especie de cierre que dificulta la llegada de la suavidad térmica y las elevadas precipitaciones características del clima atlántico. A pesar de la cercanía de Castilla y León al mar, en la parte más septentrional se localiza a tan solo $25 \mathrm{~km}$ del Mar Cantábrico, el importante cinturón que rodea a la región condiciona fuertemente las condiciones climáticas.

Estas características lo adscriben al dominio climático Mediterráneo, que afecta a la mayor parte de la extensión de los viñedos regionales, y que se caracteriza por la existencia de inviernos largos y duros con unas temperaturas medias en las llanuras centrales en el mes de enero comprendidas entre $\operatorname{los} 2$ y $\operatorname{los} 4^{\circ} \mathrm{C}$, y de veranos cortos y suaves pero con valores que superan $\operatorname{los} 22^{\circ} \mathrm{C}$ en la mayor parte del centro de la cuenca del Duero. A estos rasgos hay que añadir unos matices como son las frecuentes heladas invernales que se alargan hasta primavera (finales de abril y mediados de mayo), y que son muy perjudiciales para los viñedos.

Por otro lado las precipitaciones rondan entre los 400-450 $\mathrm{mm}$ en las llanuras del interior, frente a los más de $1.000 \mathrm{~mm}$ del reborde montañoso, repartidos entre los 
meses de otoño y primavera, dando lugar a una aridez estival bien definida que se prolonga de 3 a 4 meses entre junio y septiembre en los sectores centrales de la región. Al mismo tiempo, junto a estos imperativos climáticos, hay que añadir los microclimáticos en determinadas comarcas vitívínicolas como las Arribes (Molinero, 2007) o El Bierzo (Alonso, 2003), enclaves geográficos que presentan una altitud menor y unas temperaturas más suaves que también van a permitir el correcto desarrollo vegetativo de este tipo de cultivo.

Al clima hay que sumar las características pedológicas y agronómicas que poseen los suelos para que todo ello en su conjunto permitan obtener frutos de calidad. Para ello hay que tener en cuenta el plano superficial y la profundidad susceptible de explotación que poseen las raíces, al mismo tiempo que tampoco puede hacerse fuera de un contexto geológico y geográfico, por lo que hay que considerar los elementos que forman el paisaje en el que se enmarca, así como las distorsiones que introducen los viticultores y la propia planta.

En el caso de Castilla y León la situación de los viñedos ha variado dependiendo de la coyuntura económica del momento, aunque en numerosas ocasiones, ha estado asociada a la presencia de suelos marginales donde el desarrollo de otro tipo de cultivos era muy difícil. En la mayor parte del territorio los suelos de los viñedos se caracterizan por ser pardos sobre depósitos pedregrosos y suelos calizos sobre materiales blandos. Suelen ser profundos y poseer una buena capacidad de retención hídrica para que las raíces, cuando penetren por los horizontes, puedan captar los nutrientes necesarios que les permitan desarrollarse.

Los suelos tradicionales donde se localizaban los viñedos, de gravas y arenas, tenían un espesor superior a los $50 \mathrm{~cm}$, y en ocasiones llegaban hasta el metro, lo que hacía imposible otro tipo de labor que no fuese la viticultura. Las raíces de los cereales, como el trigo o la cebada, no conseguían penetrar hasta las capas más arcillosas por lo que no podían obtener alimento. Sin embargo, las raíces de las cepas, que cuentan con un sistema radicular más evolucionado, podían penetrar en esta capa y obtener los nutrientes necesarios para su mantenimiento. Estos suelos tienen la capacidad de retener el agua que posteriormente transfieren a la vid durante el periodo seco estival, y también son capaces de acumular calor que remitirán a la planta para obtener una buena maduración del fruto. Son suelos muy heterógeneos, sueltos y arenosos que predominan en el entorno del Duero y de sus principales afluentes como el Pisuerga, el Esla o el Arlanza, arrastrados por estos ríos, y depositados en los principales valles, vegas fluviales, terrazas y campiñas (Molinero, 1988) (Figura 1). 
Figura 1. Distribución del viñedo en Castilla y León.

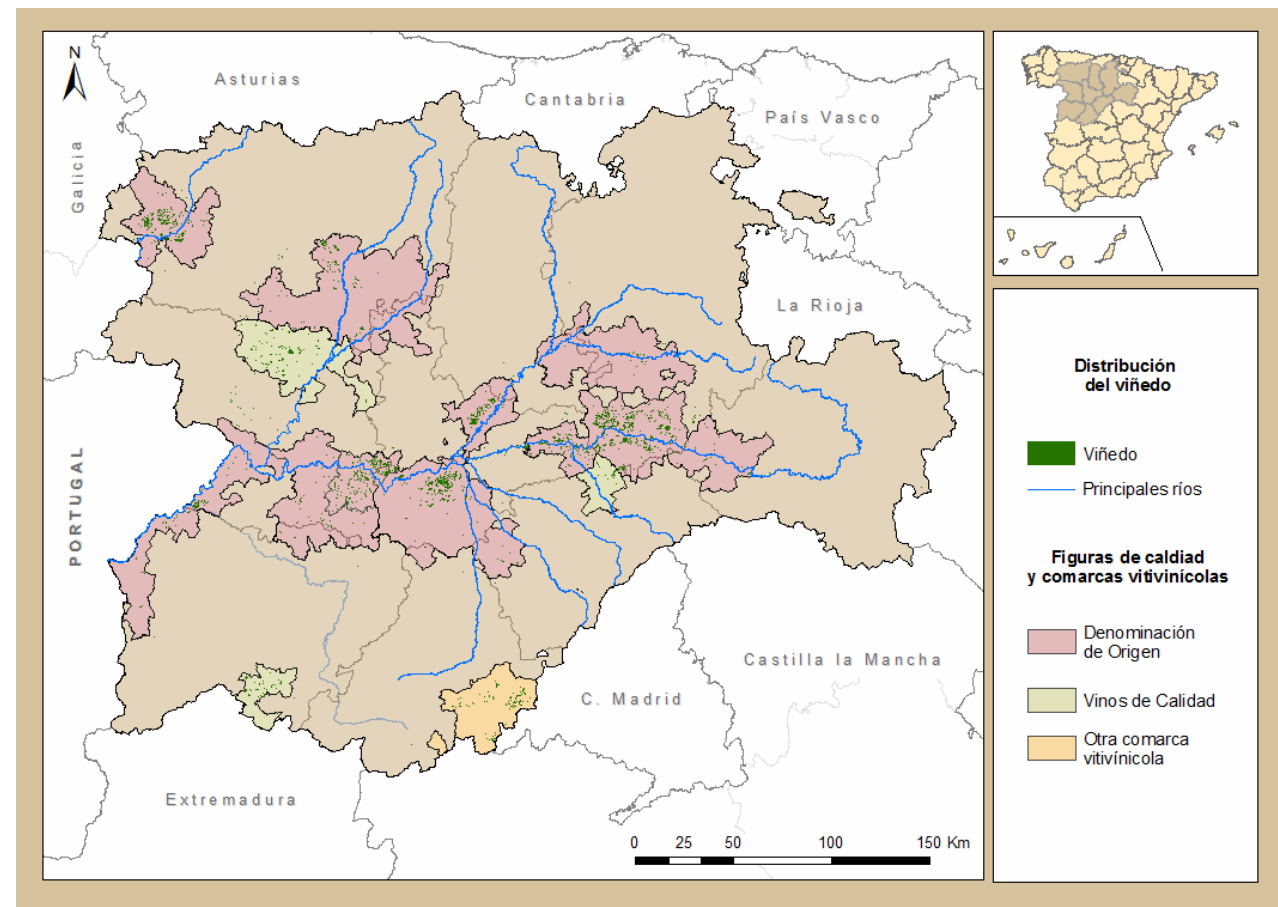

Fuente: Sistema de Información Sobre Ocupación del Suelo en España (SIOSE) 2005. Elaboración propia.

Sin embargo, a causa de la extensión de la región, existen particularidades en los materiales de los suelos dependiendo del lugar en el que se encuentren, así pues, al norte de las campiñas del Duero se localizan los páramos de Torozos y el Cerrato donde predominan los suelos calcáreos, la Tierra de Campos más arcillosa, o los páramos detríticos leoneses y algunas terrazas del Pisuerga compuestas por arenas, gravas y cantos rodados.

Estas diferencias se concretan aún más en la estructura del suelo de las comarcas vitivinícolas de la región. Por poner un ejemplo, en el entorno de la Denominación de Origen Toro los viñedos se asientan sobre sedimentos de areniscas, arcillas y pudignas calizas pliocénas que se alternan con materiales limosos y areniscas de grano grueso y fino que se han formado a lo largo del mioceno, dando lugar en la superficie a suelos pardos calizos. Sin embargo, los viñedos del Bierzo, que también son de tierra parda húmeda, están formados por una mezcla de elementos finos, cuarcitas y pizarras que provienen de las montañas que circundan a la depresión. El suelo de los viñedos de Cigales está formado por sedimentos del terciario y del cuaternario, y poseen arenas, calizas y gredas yesíferas, asentadas sobre arcillas y 
margas, y en algunas ocasiones sobre arenas, y sin embargo en la Ribera de Duero se localizan en capas más o menos lenticulares de arenas limosas o arcillosas alternándose en algunos sectores con calizas y margas.

Teniendo en cuenta todos estos elementos, la distribución espacial de la superficie de viñedo en Castilla y León va a estar condicionada por diversos factores como el clima y el suelo, pero también, como veremos en las siguientes páginas de manera más detallada, van a influir de forma clara la altitud, y en menor medida, en el caso de esta región, la pendiente y la orientación. Junto a ellos se tienen que tener en cuenta factores sociales y económicos, siendo en la actualidad uno de los más importantes la expansión de este cultivo por aquellos territorios que cuentan con alguna figura de calidad, la mayoría de ellas con Denominaciones de Origen.

\section{FUENTES DE INFORMACIÓN Y MÉTODOS EMPLEADOS}

Para la realización de esta investigación se ha utilizado la información proporcionada por el Sistema de Información Sobre Ocupación del Suelo en España (SIOSE) en 2005 extrayendo los datos pertenecientes a las parcelas de viñedos. También se ha utilizado el Modelo Digital del Terreno (MDT) de Castilla y León del cual se han obtenido los mapas concernientes a la altitud y pendiente, utilizando para la elaboración de toda la cartografía que se presenta en estas páginas el software ArcGIS (ArcMap) versión 10.1.

A continuación, se ha procedido a combinar la información del SIOSE, es decir, la capa de viñedos, con los diferentes mapas obtenidos a través del MDT, altitud y pendientes, con el objetivo de ver cómo se distribuye la superficie de viñedo atendiendo a las diferentes variables topográficas antes mencionadas.

Para la elaboración de los gráficos que se presentan en este estudio se ha utilizado la hoja de cálculo Excel. Este programa ha permitido trabajar con una cantidad importante de registros de toda la región, los cuales han sido clasificados para poder interpretarlos mejor, y facilitar, al mismo tiempo, la elaboración de los correspondientes gráficos de barras en los que se pueden ver el reparto de las vides, en diferentes intervalos, que contibuyen a explicar la distribución de la superficie en Castilla y León teniendo en cuenta estas variables.

\section{RESULTADOS. EL VALOR DE LAS VARIABLES TOPOGRÁFICAS EN LA DISTRIBUCIÓN DEL VIÑEDO}

\subsection{LA ALTITUD}

La distribución del viñedo en Castilla y León se va a caracterizar por su ubicación a lo largo del curso del río Duero y de sus principales afluentes. A medida que avanzamos desde el centro de las llanuras de esta región hacia el rolde montañoso que la rodea, el viñedo va ir perdiendo representatividad y su presencia sobre el territorio va ser cada vez menos frecuente. Este hecho va a estar condicionado por un factor 
clave en primer lugar como es la altitud, ya que a medida que esta variable aumente, la presencia de esta planta va a ser cada vez menos frecuente como consecuencia, sobre todo, del empeoramiento de las condiciones climáticas que van a dificultur el crecimiento de la misma (Figura 2).

Lo habitual es que los viñedos se ubiquen entre los 700 y los 900 metros, por lo que en este intervalo de 200 metros se van a concentrar el $71,7 \%$ de la superficie total, y si lo ampliamos añadiendo aquellos viñedos que se encuentran entre $600 \mathrm{y}$ 700 metros, esta cifra se incrementa hasta alcanzar el 83,3\% del total (Figura 3), una altitud algo superior a otros viñedos presentes en otros territorios nacionales como los de la comarca de Ocaña en la provincia de Toledo que se encuentran entre 700-720 metros de altitud (Pérez López, 1988), y muy por encima de los de algunas grandes regiones vitivinícolas vecinas como La Rioja que se encuentra en torno a los 450-500 metros (Arnáez et al, 2006) ocupando los sectores correspondientes a la depresión del Ebro en el tramo riojano y en el de la Rioja Alavesa.

Figura 2. Distribución del viñedo en Castilla y León según su altitud.

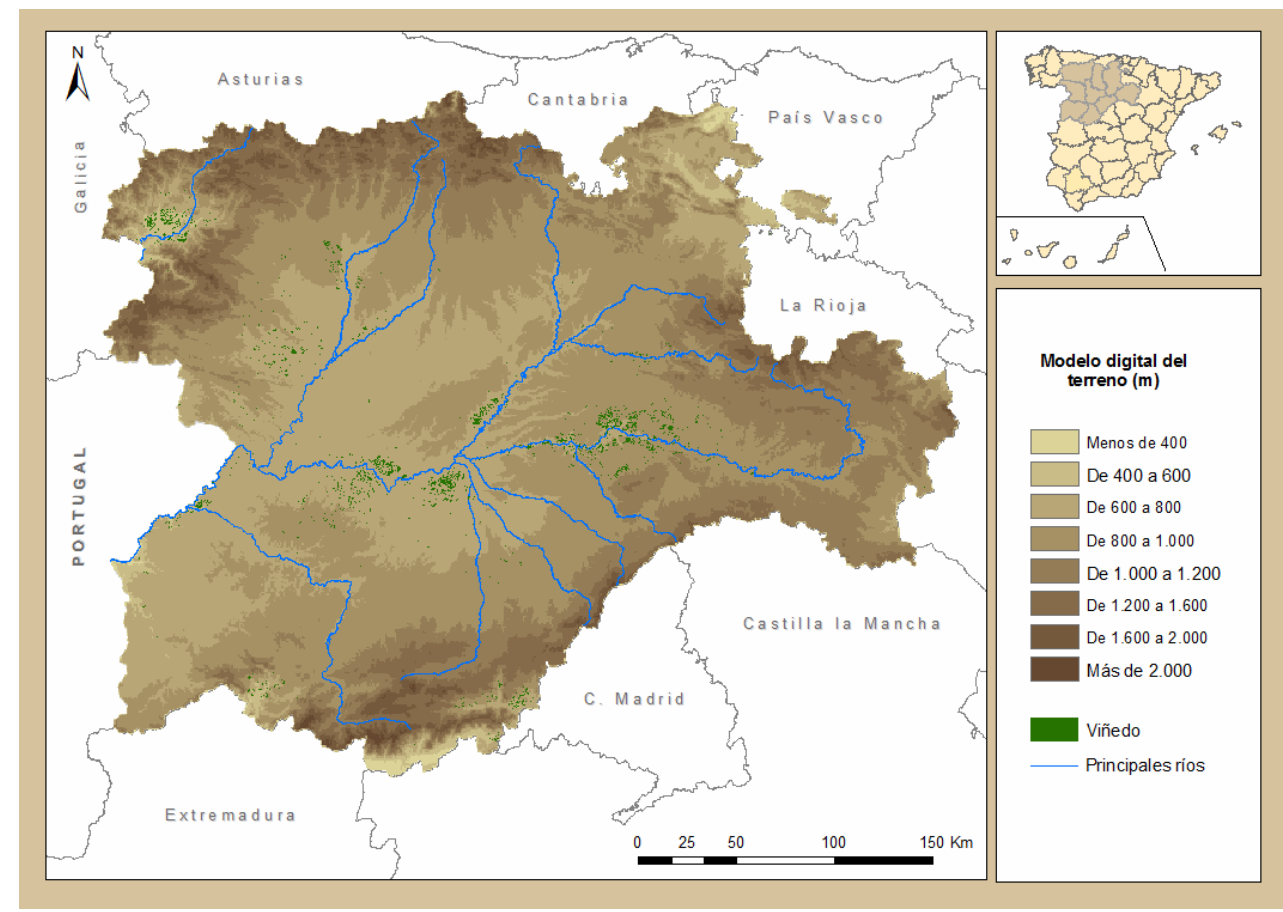

Fuente: SIOSE 2005 y MDT de Castilla y León. Elaboración propia. 
Figura 3. Distribución en porcentaje de la superficie de viñedo en Castilla y León según su altitud.

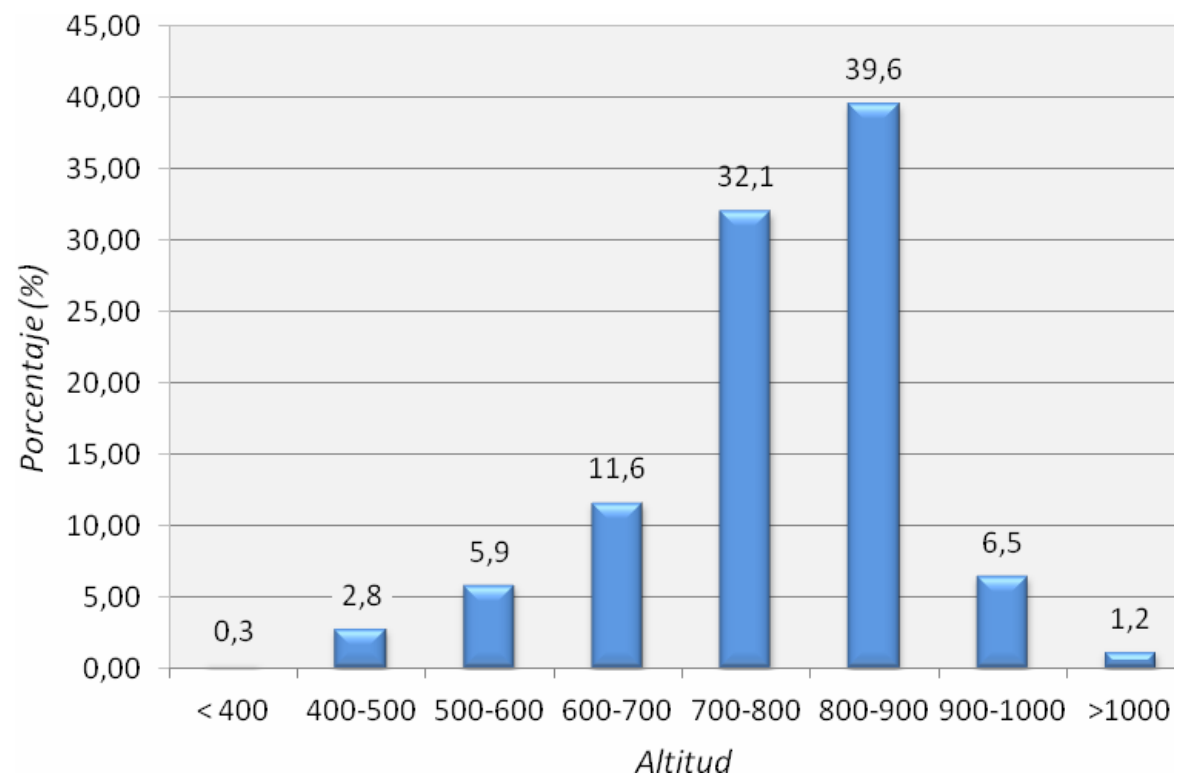

Fuente: SIOSE 2005 y MDT de Castilla y León. Elaboración propia.

Este emplazamiento se va a corresponder con las explotaciones ubicadas en la mayor parte de los municipios de las llanuras centrales del Duero aprovechando unas determinadas formas de relieve como van a ser los glacis y las terrazas fluviales del Duero, así como algunas laderas de los páramos, pero también del Pisuerga, el Arlanza y el Esla, lo que a va a dar lugar a la existencia de explotaciones de mayor tamaño que las presentes en otras comarcas vitivinícolas al no encontrar grandes obstáculos en el relieve (Figura 4).

En el caso de la Ribera del Duero destacan en el sector burgalés el municipio de Roa con 1.398 hectáreas, Aranda de Duero con 1.309, Gumiel del Mercado 1.236 y Pedrosa de Duero con 1.095 hectáreas, mientras que en el espacio vallisoletano sobresalen Pesquera de Duero y Peñafiel con 918 y 901 hectáreas respectivamente. Todas ellas localidades con un peso histórico muy representativo en el cultivo de la uva y en la producción de vino, especialmente tinto, que han vivido un boom en la expansión de las plantaciones por todo tipo de terrazgos, así como en la aparición de nuevas bodegas desde su nombramiento como Denominación de Origen en 1982. Una situación que ha permitido consolidar a esta actividad económica como un pilar clave en el desarrollo económico y rural de estos territorios; en la comarca de Rueda y su entorno despuntan La Seca con 2.800 hectáreas, Rueda con 1.390 y Serrada con 942, 
los tres núcleos más emblemáticos de la antigua Tierra de Medina, y que en las últimas dos décadas han experimentado un notable crecimiento orientando su producción hacia los vinos blancos de calidad, de variedad Verdejo; en el caso de la comarca de Toro la mayor parte de las vides se concentran en la localidad que da nombre a este espacio, es decir, en Toro, el cual presenta 2.877 hectáreas, el municipio de Castilla y León con mayor superficie superando al conjunto existente en algunas comarcas vitivinícolas como Arribes o Arlanza. En este sector zamorano también hay que señalar a Morales de Toro que alcanza las 1.373 hectáreas, siendo estas dos localidades el corazón de este espacio vitivinícola con una clara dominancia de la variedad Tinta de Toro; finalmente, en la comarca de Cigales, despuntan los municipios de Cigales seguido de Mucientes y Cubillas de Santa Marta con 1.065, 538 y 506 hectáreas respectivamente a lo largo de la margen derecha del Bajo Valle del Pisuerga, cifras inferiores a los territorios anteriores, pero que como ha ocurrido en ellos, sustentan las bases de sus correspondientes espacios de producción, en esta ocasión basado en los vinos rosados o claretes. (Todos ellos, datos procedentes de los Documentos $1 \mathrm{~T}$ de la Junta de Castilla y León en 2008).

Figura 4. Viñedos en un glacis en la localdiad zamorana de Toro.

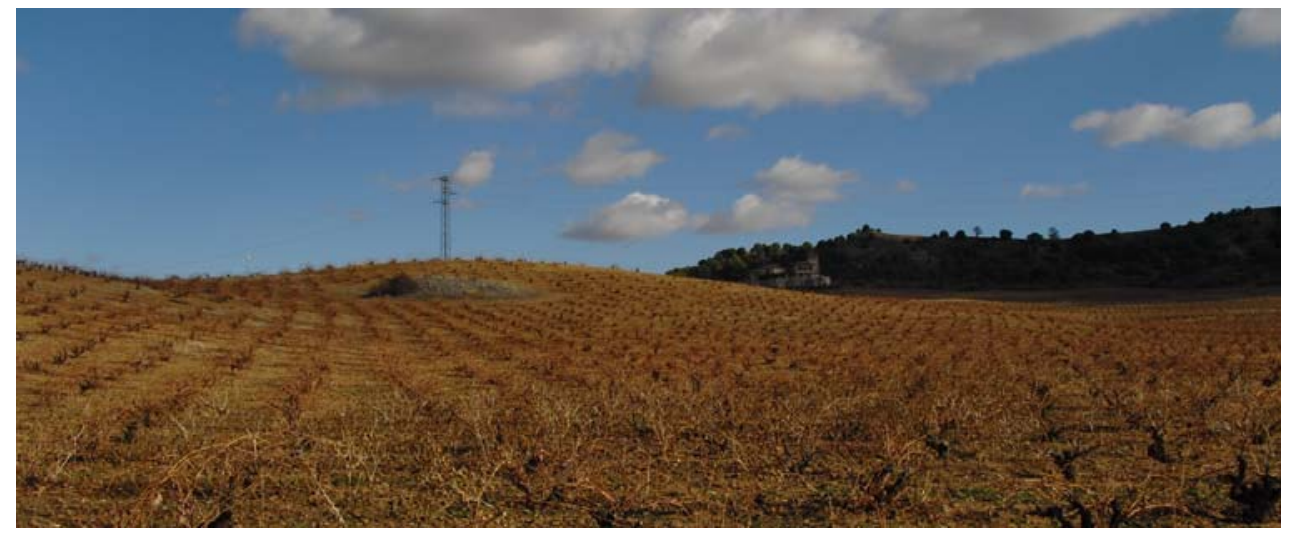

Fuente: Elaboración propia (Diciembre de 2010).

La restante superficie de vides se va a repartir, por un lado, en sectores con una altitud comprendida entre los 400 y los 600 metros, concentrando el 8,7\% del total, en espacios que van a contar con unas características climáticas específicas, diversas al resto de la Comunidad Autónoma, al presentar unas temperaturas más suaves y un menor riesgo de heladas en los meses de abril y mayo, así como en septiembre, lo que va a favorecer el cultivo de la vid, y de otros leñosos como los olivos y los árboles frutales.

En esta ocasión se correponden con los viñedos localizados en otra de las comarcas más emblemáticas de la región, la del Bierzo, y en concreto en la fosa berciana en entidades de población como Ponferrada con 1.051 hectáreas o 
Villafranca del Bierzo con 783, contribuyendo a la consolidación de esta comarca, la única reconocida legalmente como tal en Castilla y León, como un espacio productor de alimentos de calidad de muy variada índole abarcado los pimientos, las castañas, las peras, las manzanas, y como no podía ser, los vinos, todos ellos amparados bajo variadas figuras de calidad.

En estas mismas altitudes también se encuentran los viñedos de las Arribes del Duero (Figura 5) en la frontera entre España y Portugal conformando un paisaje singular de bancales, diverso a los existentes en las llanuras centrales del Duero, y que se asemeja a espacios como los de la región de la Liguria en Italia, y en concreto de Le Cinque Terre, donde los viñedos se combinan junto a los frutales y los olivos en diferentes niveles teniendo en cuenta la disposición del relieve. En este espacio destaca la localidad de Fermoselle, que se consolida como el núcleo más representativo con una superficie de 1.546 hectáreas.

Figura 5. Viñedos abancalados en Fermoselle conviviendo con otros cultivos.

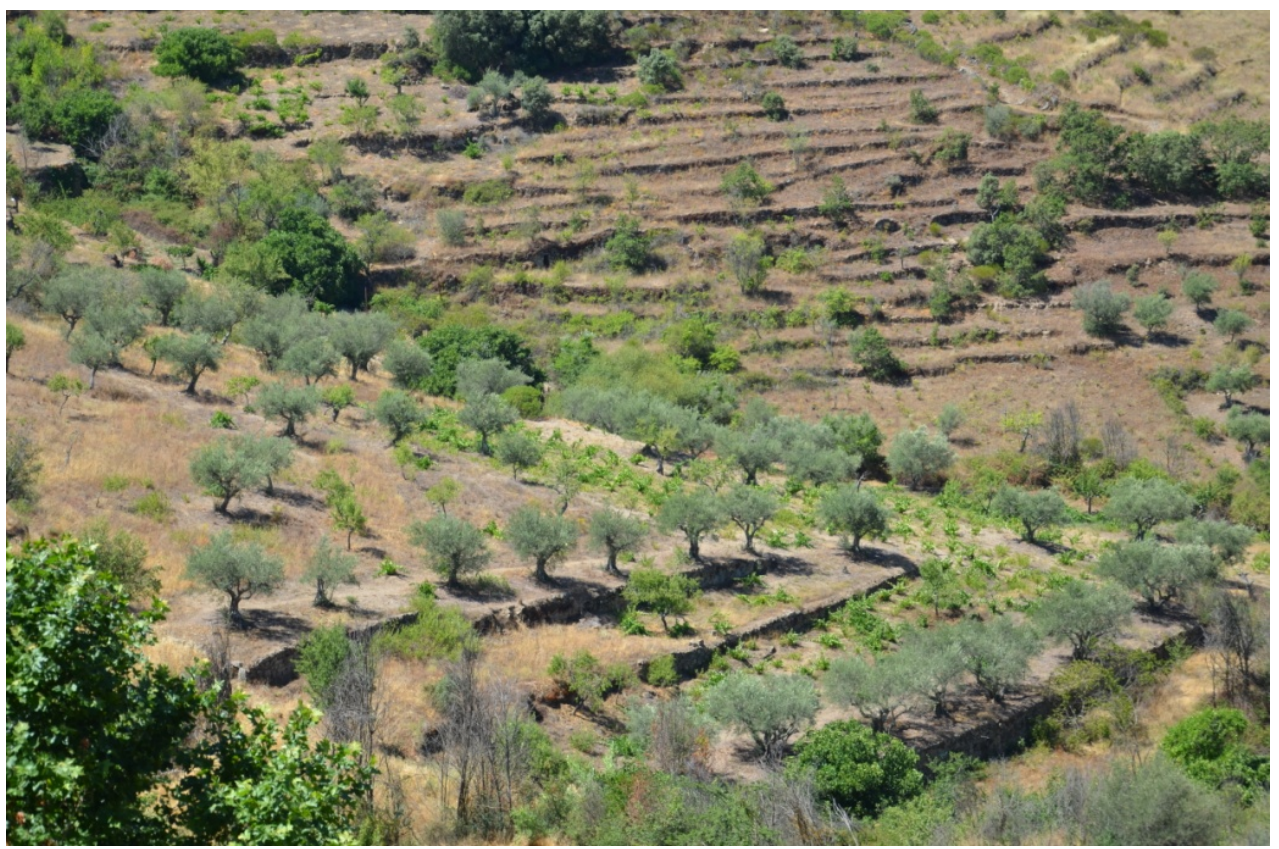

Fuente: Elaboración propia (Julio de 2012). 
Junto al Bierzo y las Arribes hay que mencionar el sector de Cebreros y la Sierra de Salamanca, todos ellos espacios vitivinícolas, unos con mayor densidad que otros, pero que presentan unos rasgos físicos específicos con características propias, lo que al mismo tiempo les confiere cierta entidad respecto a otros territorios de la región, aunque no llegan a alcanzar el reconocimiento de los espacios vitivinícolas del centro de las llanuras.

Por debajo de los 400 metros la superficie existente en Castilla y León es muy escasa $(0,3 \%)$, reduciéndose a unos pequeños enclaves en el sur de Ávila en determinados sectores del entorno de Cebreros, así como en el noroeste de Burgos en el territorio denominado Chacolí-Merindades.

Situación muy similar sucede a medida que se avanza hacia el cíngulo montañoso que rodea la región, donde la presencia de este cultivo va desapareciendo como consecuencia del empeoramiento de las condiciones climáticas, y su existencia es casi testimonial, ya que la rigurosidad del clima impide el correcto desarrollo de la planta. Los viñedos localizados a una altitud superior a 900 metros $(7,7 \%$ del total $)$ se emplazan en algunos puntos de la Ribera del Duero soriana como en Carrascosa de Abajo, Recuerda o Villanueva de Gormaz, así como en los sectores más septentrionales de la Ribera del Arlanza. A partir de los 1.100 metros de altitud la vid encuentra su límite ecológico en Castilla y León, y desaparece dejando paso a otros cultivos más aptos para este tipo de espacios.

\subsection{LA PENDIENTE}

La pendiente es otro de los aspectos que va a marcar la distribución de los viñedos en la región (Figura 6). En el caso de Castilla y León la pendiente media se sitúa en torno al 2,0\%, una cifra muy baja y bastante inferior si lo comparamos con los viñedos riojanos que son del 18,7\% (Arnáez et al., 2006), unos valores que van a traer consigo importantes problemas de erosión sobre el terrazgo como consecuencia de algunos eventos lluviosos de alta intensidad y baja frecuencia que dan lugar a la formación de surcos, cárcavas y áreas de sedimentación (Ruiz Flaño et al., 2008).

El $77,5 \%$ del viñedo regional se sitúa por debajo del $5 \%$ de pendiente, cifra que se incrementa en un $15,5 \%$ más si se tiene en cuenta el terrazgo con una pendiente comprendida entre el 5 y el $10 \%$, lo que entre ambos, dan como resultado que, el $93 \%$ de las vides de esta región se localicen por debajo del 10\% (Figura 7), valores que muestran una de las características básicas del centro de la cuenca del Duero como es la planitud. Una particularidad que ha sido recogida por viajeros y estudiosos que han recorrido y atravesado estas tierras como Richard Ford, el cual se refería al interior de ambas castillas y de La Mancha como un espacio que posee "extensas e indefensas llanuras" (1974: 23), o Humboldt, que a su entrada en Castilla por el País Vasco describe la diferencia entre ambos territorios "Tan pronto se entra en Castilla, el contraste con la Bella Vizcaya es horrísono. Campiña pelada la llaman de manera muy expresiva los españoles: en lejanía, algunas peladas montañas y nada más." (1998: 59). Características que han contribuido a consolidar una imagen del interior 
de Castilla fiel a la realidad, como un emblema propio y un rasgo distintivo que se reflejan de forma clara y contundente en la siguiente cita:

La llanura se extendía monótona, desnuda, terrosa, bajo un cielo no menos indefinido y escueto. No se podía decir si punzaba más en la piel el frío del ambiente, ó en el alma la desnudez de todas las lejanías: planicie que daba ganas de pensar en un astro desalquilado. Sólo se descubrían por todas partes cavones revueltos por el arado. Ni un árbol, ni una zarza, ni un tono verde. Un tinte amarillo sucio con degradaciones grises era la única coloración que manchaba la extensión sin límites. En general las tierras aradas se mostraban limpias, cual si sus terrones simbolizasen la propia esterilidad. La población vegetal delatábase tan sólo por rastrojos pajizos, cardos secos ó gatuñas rastreras; la animal, por tal cual alondra que proyectaba sus postreros vuelos sobre el horizonte; la humana..., por un jinete que penosamente se adelantaba campo a traviesa entre aquellas tierras desoladas. (Macías Picavea, 2006: 11).

Figura 6. Distribución del viñedo en Castilla y León según su pendiente.

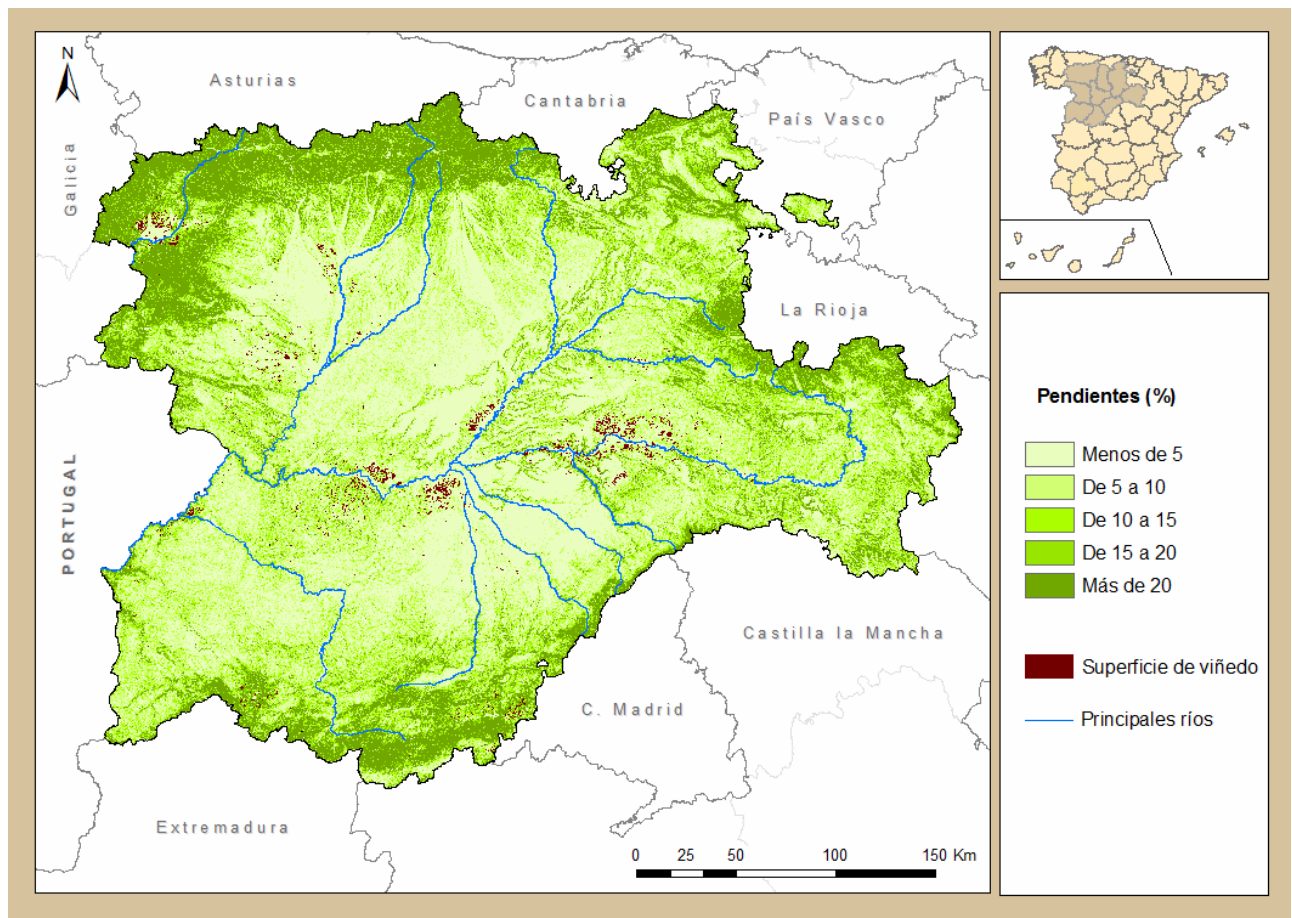

Fuente: SIOSE 2005 y MDT de Castilla y León. Elaboración propia. 
Figura 7. Distribución en porcentaje de la superficie de viñedo en Castilla y León según su pendiente.

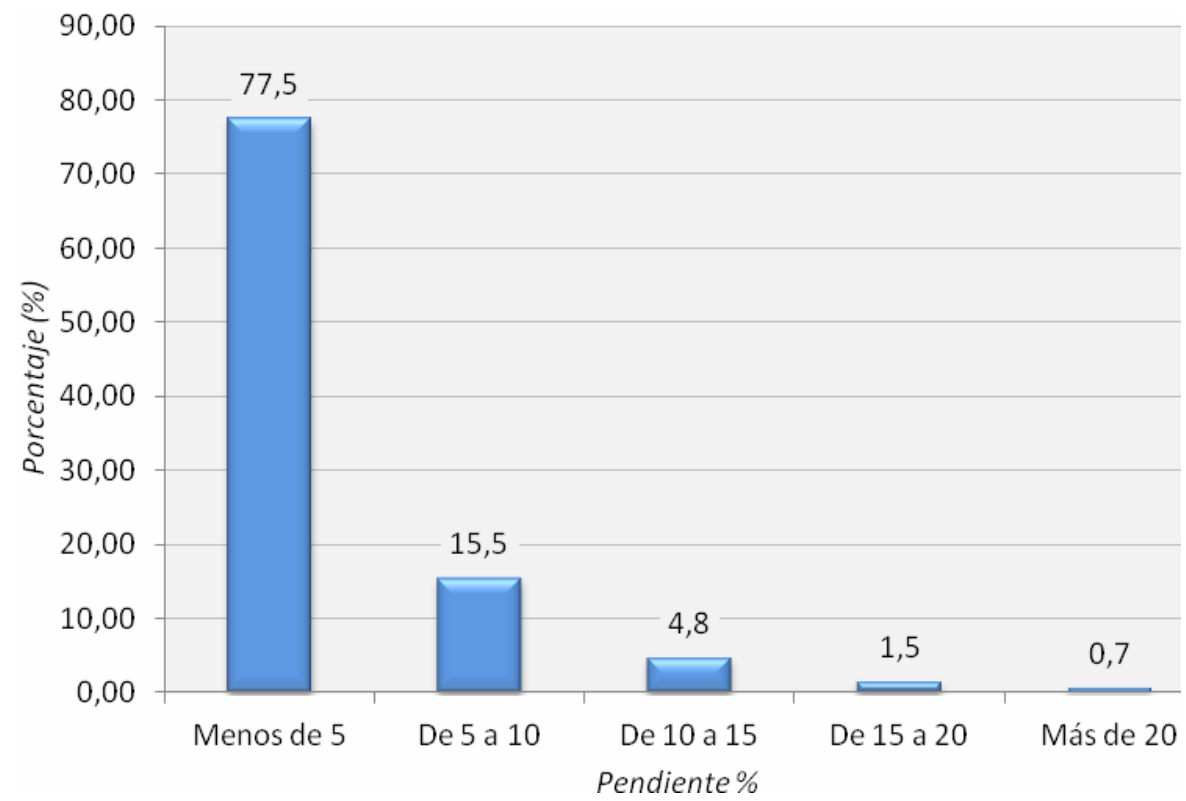

Fuente: SIOSE 2005 y MDT de Castilla y León. Elaboración propia.

La distribución de estos viñedos con este tipo de pendiente, inferior al $10 \%$, se van a corresponder con la mayor parte de la superficie existente, es decir, con la práctica totalidad de las comarcas vitivinícolas de Rueda, Ribera del Duero, Cigales, Toro, Tierra de León o Tierra del Vino de Zamora, sectores prácticamente llanos (Figura 8). Espacios con horizontes amplios y ondulaciones suaves donde resulta complicado vislumbrar el infinito resultado de la planitud como ocurre en Toro (Sánchez Hernández, 2003), pero que se pueden extrapolar al resto de territorios, ya que todos ellos presentan unas características muy similares entre sí.

Este tipo de emplazamientos permite realizar de forma más cómoda las tareas derivadas del cultivo de la vid, pero sobre todo ha facilitado la mecanización de algunas de ellas. La introducción de tractores para arar el terrazgo y de máquinas vendimiadoras para recoger la uva, han permitido ahorrar costes y tiempo a los viticultores, a la vez que ha facilitado el trabajo en las viñas generalizándose en determinados espacios, sobre todo en Rueda donde la práctica totalidad de la vendimia se lleva a cabo con estas grandes máquinas. Un proceso que se ha visto apoyado en una serie de transformaciones que se han producido en el paisaje de la vid tradicional, y en concreto en la estructura del terrazgo, al pasar en unos sectores determinados, de pequeñas e irregulares parcelas esparcidas por el territorio, a 
explotaciones más uniformes y de mayor tamaño, algunas de ellas fruto de la concentración parcelaria, o por otro lado, gracias a la adquisición por parte de medianas y grandes bodegas de determinados pagos de viñedos, aledaños entre sí, pertenecientes a pequeños propietarios que han decidido venderlos; también hay que tener en cuenta los cambios en los sistemas de plantación, al pasar de cepas en vaso a otras en espaldera, donde las alambres se constituyen como un elemento más del terruño que se encarga de guiar a la cepa durante su crecimiento; o un incremento en la densidad de plantación de los majuelos con un mayor número de vides por hectárea.

Figura 8. Viñedos en el término municipal de La Seca.

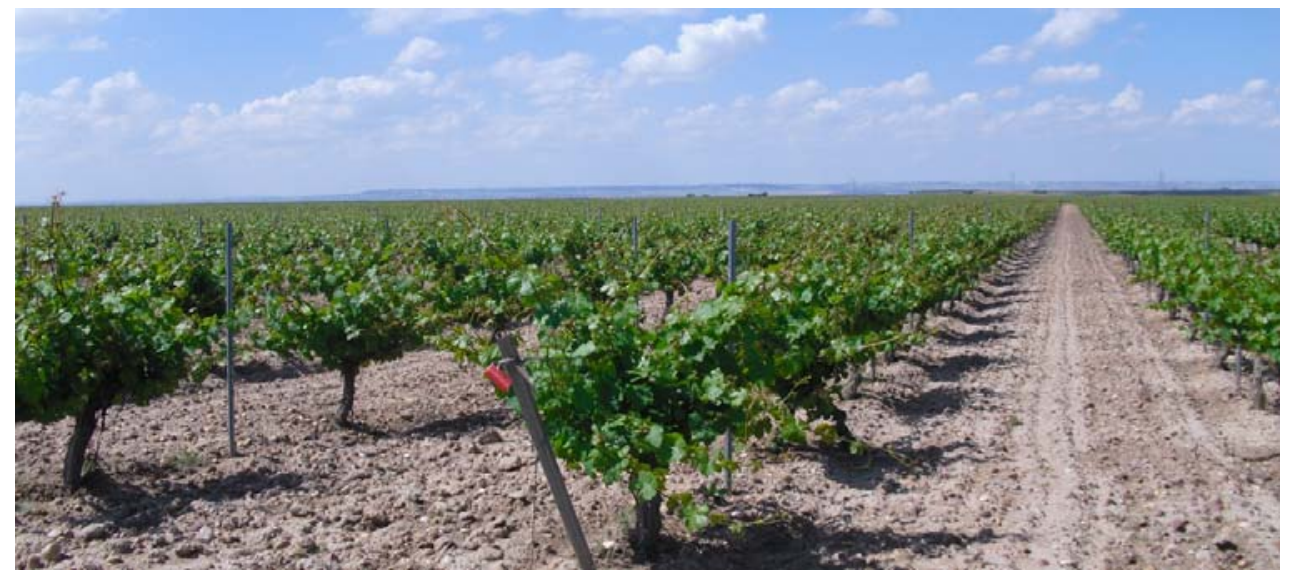

Fuente: Elaboración propia (Mayo de 2011).

No obstante, en determinados sectores de los espacios vitivinícolas anteriormente mencionados, y en cocreto en la Ribera del Duero, el viñedo se expande en terrazgos con una pendiente mayor, algo superior al $10 \%$, enlazando las explotaciones ubicadas en los sectores más planos con el comienzo de las laderas de algunos páramos (Figura 9). El boom de las plantaciones acaecido desde mediados de la década de los noventa del siglo XX propicició la expansión de la vid por todo tipo de terrazgos, aprovechando diversas clases de suelos y formas del relieve para poder hacer frente a la nueva demanda del mercado. 
Figura 9. Viñedos en el término municipal de Peñafiel.

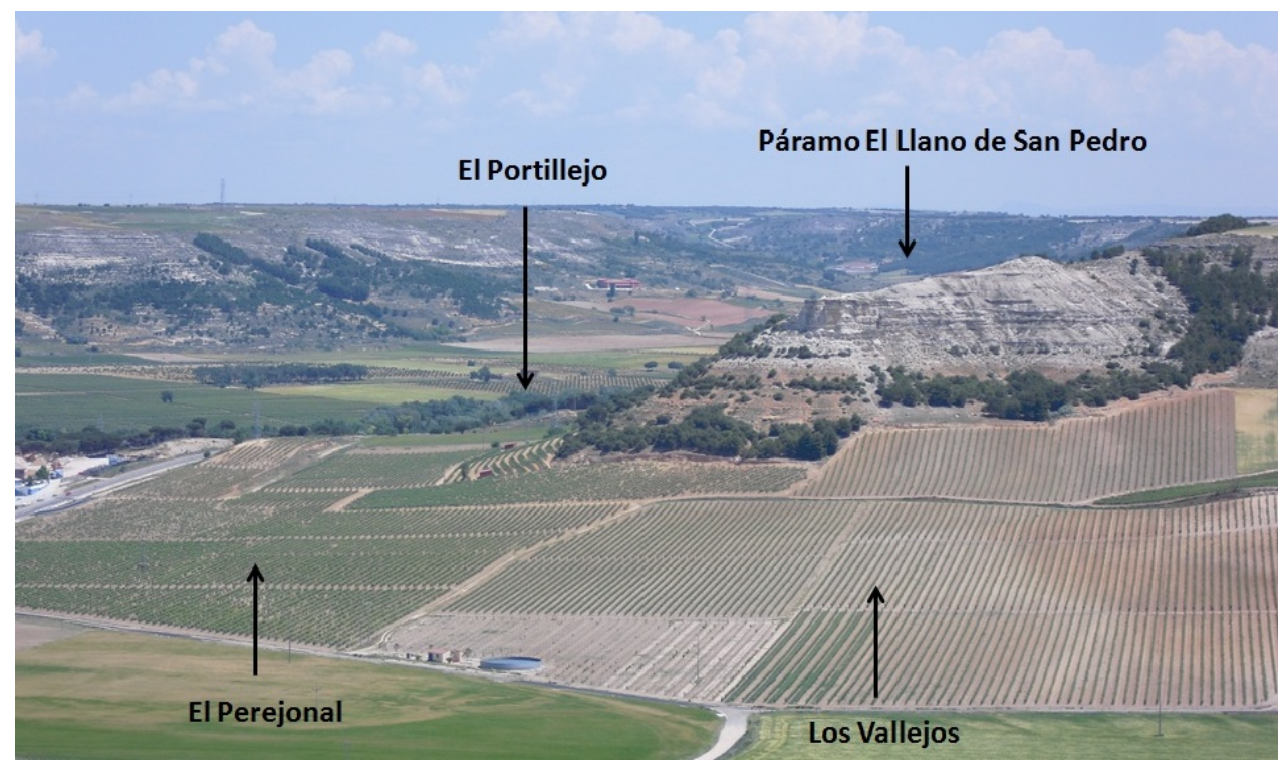

Fuente: Elaboración propia (Junio de 2011).

Sin embargo, es en las comarcas del Bierzo y de las Arribes donde la pendiente de este cultivo es mayor, entre el 10 y el $20 \%$ y más del $20 \%$, representando en su conjunto tan sólo el $6,9 \%$ y el $0,7 \%$ respectivamente de la superficie total, pero que en estas comarcas vitivinícolas es muy propio debido a su conformación física, diversa a la existente en la cuenca del Duero. En el caso del Bierzo se van a localizar en las terrazas y laderas del entorno de la Hoya del Bierzo en los cursos bajos de los ríos Sil, Boeza, Oza, Cúa y Burbia, y que, a pesar de esta pendiente, no se han abancalado (Alonso Santos, 2003), mientras que en las Arribes van a aprovechar el sistema de bancales para el desarrollo de este cultivo, pudiendo salvar de esta forma las gargantas existentes creadas por el río Duero en estas penillanuras.

\subsection{LA EXPOSICIÓN}

Finalmente, la tercera variable empleada para la realización de este estudio es la exposición de los viñedos. El resultado de la localización de este cultivo según este elemento va a tener en cuenta los otros dos analizados anteriormente, la altitud y la pendiente. Se ha visto como la ubicación del viñedo en Castilla y León se concentra, en su mayor parte, en las llanuras centrales del Duero en una altitud más o menos homogénea, sin grandes contrastes, dando lugar a una escasa pendiente, utilizando los sectores más llanos. El aprovechamiento de este tipo de terrazgo va a determinar el tipo de exposición predominante, pero que, al ser prácticamente llano, esta variable va a poseer un peso muy poco representativo en Castilla y León. 
A pesar de todo, domina la exposición meridional con un 42,29\% (Figura 10), una cifra similar a la La Rioja en esta misma exposición que es del 42,2\% (Arnáez et al. 2006). Esta opción es la más habitual a la hora de plantar vides en cualquier territorio, ya que este tipo de planta necesita un importante número de horas de sol al año (unas 2.500), sobre todo durante la maduración del fruto, por lo que es vital una buena orientación que permita a la planta recibir los rayos del sol que la permitan conseguir su correcto desarrollo vegetativo.

Figura 10. Distribución en porcentaje de la superficie de viñedo en Castilla y León según su exposición.

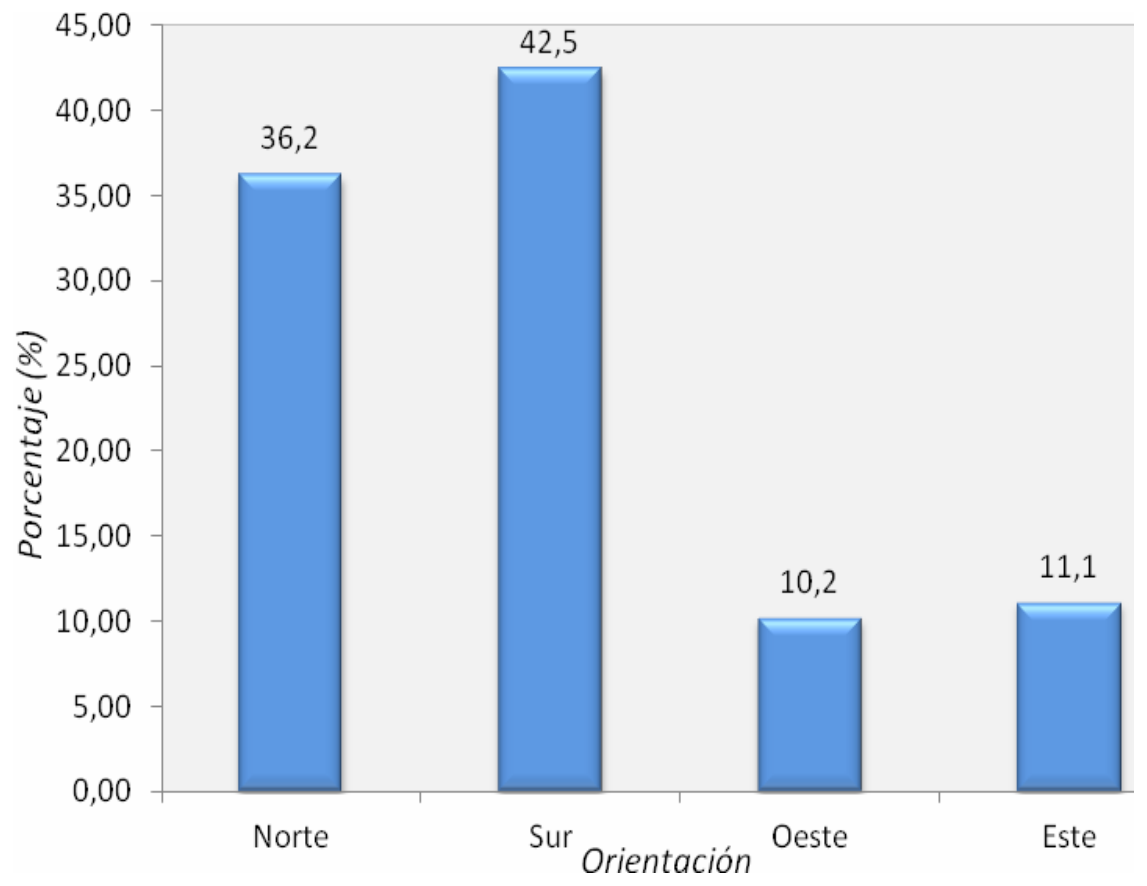

Fuente: SIOSE 2005 y MDT de Castilla y León. Elaboración propia.

Sin embargo, en Castilla y León, y como ya se ha explicado, a causa de la planitud de las llanuras centrales de la cuenca del Duero, el viñedo se va a expandir por todo tipo de territorios con diversas exposiciones. Así pues, y aunque la primera opción sea la meridional, la septentrional adquiere un peso muy significativo alcanzando el $36,02 \%$, cifra superior si lo volvemos a comparar de nuevo con el estudio de Arnáez et al. de La Rioja, donde los viñedos de esta comarca vitivinícola, en esta exposición norte, representan el $25,3 \%$ del total como consecuencia de la presencia de un 
territorio más accidentado, que va a repercutir en que los viticultores planten sus vides en sectores donde los rayos del sol tengan más incidencia.

En las llanuras del Duero, una exposición septentrional no implica una orientación exclusiva a la umbría como podría suceder en otros espacios con mayor pendiente como en los casos europeos de los viñedos del Piamonte italiano o los del Lavaux en Suiza, sino que, al ser un relieve prácticamente llano con suaves ondulaciones y con escasa pendiente, da lugar a un espacio más abierto que permite la recepción de los rayos del sol en las cepas sin ningún problema. Es decir, amplias planicies propiamente castellanas, y que son una constante en la obra de numerosos autores que han trabajado sobre Castilla y León, y han descrito con acierto una parte del paisaje inconfundible del interior de esta región. Un ejemplo muy significativo lo encontramos en la obra de Macías Picavea, escritor del Regeneracionismo afincado en Valladolid, y que fue Catedrático de Instituto del José Zorilla impartiendo las asignaturas de Latín y Geografía. Este autor era un excelente conocedor de los pueblos, de las las personas y de la economía de Castilla, y prueba de ello se refleja en las descripciones que realizó de algunas de sus comarcas, como por ejemplo de la Tierra de Campos con las siguientes palabras "La llanura se extendía monótona, desnuda, terrosa, bajo un cielo no menos indefinido y escueto" (2006: 11).

Una idea que se va a repetir también De Figueroa y Melgar en el viaje que hizo de Burgos a Madrid pasando por Valladolid en 1840, el cual relata sobre los campos de Castilla lo siguiente "La comarca que atravesábamos tenía un aspecto extrañamente salvaje: llanuras inmensas, áridas, sin un solo árbol que rompiese su monotonía, terminadas en montañas de un amarillo ocre, a las cuales ni la lejanía lograba dar un tinte azulado." (1971: 244).

Finalmente, Humboldt describe lo mismo que los autores anteriores, pero en esta ocasión cuando entra en Castilla a través de la provincia de Álava mostrando la dualidad existente entre sendos territorios "Poco después de Miranda, una columna con una inscripción señala los límites de Álava y Castilla. Ya casi desde Salinas, se modifica la fisonomía del paisaje; ya no hay tanta alternancia de montañas y valles y sobre todo ya no hay esa abundancia de árboles. Todo tiende y se asemeja ya al desierto y llanura castellanos, sobre todo después de Vitoria." (1998: 59).

\section{CONCLUSIONES}

Como se ha podido analizar, el relieve de Castilla y León se divide en dos unidades bien diferenciadas entre sí y con fuertes contrastes altitudinales, geomorfológicos, climáticos, biogeográficos, económicos, agrarios, etc. Estas dos unidades son los cordales montañosos que circundan prácticamente toda la región superando los 2.000 metros de altitud en determinados sectores, y que constituyen el límite físico y administrativo, y por otro lado se encuentra la cuenca sedimentaria del Duero constituida por diversas formas del relieve y con una altitud media comprendida entre los 700 y los 900 metros. En este último espacio es donde se asientan la mayor parte de la población y de las actividades económicas, entre ellas los viñedos. 
El viñedo es un cultivo clave en la economía de numerosos municipios de Castilla y León. El trabajo en los majuelos, y su posterior transformación de la uva en vino en la bodega, ha permitido diversificar la economía de un conjunto muy importante de espacios rurales de la región, no sólo en la producción de vino, sino en una serie de actividades derivadas de la elaboración de estos caldos destacando de forma clara el enoturismo. En definitiva, de aquellas localidades que presentan unas variables topográficas específicas que van a permitir el correcto desarrollo vegetativo de las vides, y con ello, de la expansión de un sector vitivinícola en la mayoría de los casos enfocados a la producción de vinos de calidad.

La distribución de los viñedos en Castilla y León sigue unas pautas determinadas, y las variables topográficas, y en concreto la altitud y la pendiente, y en menor medida la orientación, van a tener un peso muy relevante a la hora de la localización y expansión de las vides por el territorio regional.

Así pues, se ha visto en las páginas anteriores como los viñedos se emplazan, la mayor parte de ellos, en las llanuras centrales del río Duero, a lo largo de su curso, y con unas altitudes comprendidas entre los 600 y los 900 metros, concentrando la superficie de algunas comarcas vitivinícolas como la Ribera del Duero, Rueda o Toro, así como en algunos de sus afluentes como el Pisuerga y los viñedos del entorno de Cigales, del Esla en el caso de la Tierra de León, o del río Arlanza en las localidades que componen el territorio que lleva el mismo nombre. Por lo tanto, a medida que nos alejamos del curso del Duero, y con ello, se va produciendo un aumento de la altitud, la superficie de viñedo va perdiendo representatividad en el territorio a favor de otro tipo de cultivos mejor adaptados a las características climáticas específicas que van definiendo estos espacios.

Junto a la altitud hay que tener en cuenta la pendiente. En Castilla y León, la mayor parte del cultivo de la vid, en concreto el $93 \%$ del total se encuentra por debajo del 10\%, unas cifras que indican la planitud característica de las llanuras centrales del Duero, lugar donde, como ya se ha comentado, se concentra la mayor parte de la superficie de viñedo. Pendiente que se deriva de los escasos contrastes altitudinales existentes en este sector, y que van a permitir un manejo más sencillo de las explotación, así como la introducción de determinada maquinaria para la realización de algunas de las labores típicas de los viñedos como puede ser la vendimia.

Finalmente, la tercera variable topográfica analizada, la exposición, aunque es esencial tenerla en cuenta a la hora de plantar un viñedo, en el caso de Castilla y León va a a presentar un papel más reducido a causa de la planitud de sus llanuras. La escasa pendiente en los sectores de vides y la casi horizontalidad del terrazgo castellano, van a generar que este cultivo no destaque de forma exclusiva por un tipo de orientación. Si bien, es cierto, que la mayoría de ellos presentan una exposición meridional, típica y característica de este tipo de cultivos, la septentrional posee bastante relevancia frente a la que que tienen en otras comarcas vitivinicolas como La Rioja, y se encuentran en unas cifras muy cercanas a los viñedos orientados al sur.

A pesar de todo, aunque esta es la dinámica dominante y más característica de la distribución de los viñedos en la Comunidad Autónoma de Castilla y León, hay que 
mencionar que existen un conjunto de espacios que presentan una serie de singularidades a la hora de distribuir este cultivo por su territorio. Como enclaves específicos hay que destacar sobre todo el Bierzo y las Arribes, pero también otros sectores como la Sierra de Salamanca y Cebreros, todos ellos con viñedos localizados a una altitud inferior a los 600 metros, incluso en determinados sectores por debajo de los 400, al mismo tiempo que poseen una pendiente, superior al $10 \%$, más elevada que las llanuras centrales de Castilla y León. Estos sectores presentan una estructura de la tierrra más pequeña e irregular, por lo que el viñedo tiende a concentrarse en todo tipo de parcelas, de las cuales, muchas de ellas, se encuentran en bancales para poder salvar las desigualdades derivadas del relieve.

Como se ha demostrado en estas páginas, la distribución del viñedo en esta región va a estar condicionada por una serie de variables topográficas que van a limitar el cultivo de las vides teniendo en cuenta las características que presenta el territorio, así como las propias de la planta. Todo ello, aspectos que van a permitir configurar un mapa regional del viñedo que concentra la superficie de viñedo en unos territorios concretos.

\section{BIBLIOGRAFÍA}

ALARIO, M. (1991). Significado espacial y socioeconómico de la concentración parcelaria en Castilla y León. Madrid, Ministerio de Agricultura, Pesca y Alimentación.

ALONSO, J.L. (2003). Redes y procesos de innovación en las comarcas vitivinícolas de Castilla y León: el ejemplo de la D.O. Bierzo. Boletín de la Asociación de Geógrafos Españoles, 36, 43-60.

ARIAS, P. (2000). El proceso de mecanización en la actividad agrícola. Estudios Agrosociales y Pesqueros, 187, 9-38.

ARNÁEZ, J. et al. (2006). Distribución espacial del viñedo en la Comunidad Autónoma de La Rioja: influencia de la topografía y de las formas del relieve. Polígonos. Revista de Geografía, 16, 11-34.

BARAJA, E. (Coord.) (2006). Atlas de los regadíos de la Cuenca del Duero. Segovia, Universidad de Valladolid.

BLANCO, J.F. (2009). Los inicios del consumo de uva y ¿del cultivo de la vid? en Cauca vaccea. En SANZ, C. y ROMERO, F. (Eds.). El vino y el banquete en la Europa prerromana. Valladolid, Centro de Estudios Vacceos "Federico Wattenberg" de la Universidad de Valladolid, 213-224.

CAMARERO, C. (1984). A propósito de la "crisis de la filoxera": la actitud de la administración ante la invasión filoxérica y algunas precisiones estadísticas. Revista de Historia Contemporánea, 3, 99-124.

CONSEJERÍA DE AGRICULTURA Y GANADERÍA DE LA JUNTA DE CASTILLA Y LEÓN

(http://www.jcyl.es/web/jcyl/AgriculturaGanaderia/es/Plantilla100/1241431154222/_ - $)$. 
DE FUIGUEROA, A. (1971). Viajeros románticos por España. Madrid, Escuelas Profesionales Sagrado Corazón.

FORD, R. (1974). Las cosas de España. Madrid, Ediciones Turner.

GARCÍA, J. (1986). El clima en Castilla y León. Valladolid, Editorial Ámbito.

HUETZ, A. (1967). Vignobles et vins du nord-ouest de l'Espagne. Bordeaux, Institut de Géographie.

HUMBOLDT, A. (1998). Diario de viaje a España 1799-1800. Madrid, Ediciones Cátedra, S.A.

MACÍAS, R. (2006). La Tierra de Campos. Valladolid, Ayuntamiento de Valladolid y Diputación de Valladolid.

MOLINERO, F. (1988). Viñedos y vinos de Valladolid. Cuadernos Vallisoletanos, 41, $1-30$.

MOLINERO, F. (2007). Oportunidades y perspectivas de los viñedos de Las Arribes del Duero. Populaçao e Sociedade, 13, 97-106.

MOLINERO, F. (2011). Los paisajes del viñedo en Castilla y León: tradición, renovación y consolidación. Polígonos. Revista de Geografía, 21, 85-117.

MONTOYA, E. (2012). El viñedo en la historia de la agricultura burgalesa. Burgos, Publicaciones de la Excelentísima Diputación Provincial de Burgos.

MORALES, C. y ORTEGA, M.T. (2002). Síntesis del clima en Castilla y León: factores y características. En LONGARES, L.A. et al. (Coords.): Aportaciones geográficas en Memoria del Profesor L. Miguel Yetano Ruíz. Zaragoza, Servicio de Publicaciones de la Universidad de Zaragoza. 385-394.

PÉREZ, J.J. (1988). El viñedo en la comarca de Ocaña. En PILLET, F. (Coord.): El espacio rural de Castilla-La Mancha: Reunión de Estudios Regionales de CastillaLa Mancha. Ciudad Real, Diputación provincial de Ciudad Real. 279-286.

PIQUERAS, J. (2005). La filoxera en España y su difusión espacial: 1878-1926. Cuadernos de Geografía, 77, 101-136.

RUIZ, P. et al. (2008). Procesos de erosión y pérdidas de suelo tras lluvias intensas en viñedos de La Rioja (España). Zubía, 25-26, 61-73.

SÁNCHEZ, J.L. (2003). Capital exógeno y procesos de innovación en la industria vinícola de la denominación de origen Toro. Boletín de la Asociación de Geógrafos Españoles, 36, 61-79.

SANZ, C. et al. (2009): El vino y el banquete en la Ribera del Duero durante la protohistoria. Valladolid, Centro de estudios Vacceos "Federico Wattenberg" de la Universidad de Valladolid.

SOLANO, M.T. (1991). La crisis del viñedo. La filoxera en España. Madrid, Editorial de la Universidad Complutense de Madrid.

VALDEÓN, J. (2006). La reconquista. El concepto de España: unidad y diversidad. Madrid, Editorial Espasa. 